\title{
ANTICROWDING POPULATION MODELS IN SEVERAL SPACE VARIABLES
}

BY

GASTON E. HERNANDEZ

University of Connecticut, Storrs, Connecticut

1. Introduction. In this paper we discuss a model for diffusion of populations with age dependence in several space variables. The fundamental principle states that in a fixed region in space the population can change only through births, deaths, immigration, and emigration (see [12]). In a small interval of time $d t$ the change in the population $\rho$ is

$$
D \rho=(B-D+I-E) d t .
$$

1.1. Age dependent populations. Let $\rho(t, a)$ be the age population density, i.e., $\int_{a_{1}}^{a_{2}} \rho(t, a) d a$ represents the number of individuals at time $t$ of ages between $a_{1}$ and $a_{2}$. In particular $u(t)=\int_{0}^{\infty} \rho(t, a) d a$ is the total population at time $t$. A change of $h$ units in time implies a change of $h$ units in age. Thus assuming differentiability $D \rho=\rho_{t}+\rho_{a}$. When using Malthus's law for births and deaths, with $I=E=0$, eq. (1.1) becomes

$$
\rho_{t}+\rho_{a}=-\mu \rho,
$$

where $\mu$ might depend on $a$. Integrating along characteristics we arrive at the formal solution

$$
\rho(t, a)= \begin{cases}\rho(0, a-t) e^{-\int_{0}^{t} \mu(a-t+s) d s} & t \leq a, \\ \rho(t-a, 0) e^{-\int_{0}^{t} \mu(s) d s} & t \geq a .\end{cases}
$$

Thus in addition to specifying the initial age distribution $\rho(0, a)=\rho_{0}(a)$, we also need to specify $\rho(t, 0)$, the number of newborns at time $t$. Assuming that the population sex ratio remains constant, the birth rate $\beta(a)$ is defined such that $\beta(a) d a$ represents the average number of offsprings produced per unit time by an individual aged between $a$ and $a+d a$. In this way there is a birth law

$$
B(t)=\rho(t, 0)=\int_{0}^{\infty} \beta(a) \rho(t, a) d a .
$$

The Lotka-Von Foerster model (also McKendrick-Von Foerster) consists of Eqs. (1.2) and (1.4) along with a nonnegative initial condition $\rho_{0}(a) \geq 0$. In $[15,18]$ Gurtin and MacCamy proposed a model in which the birth modulus and the death 
modulus depend also on the total population $u(t)$. The Gurtin-MacCamy model for age dependent populations without diffusion is:

$$
\begin{aligned}
& \rho_{t}+\rho_{a}=-\mu(a, u) \rho, \\
& \rho(t, 0)=B(t)=\int_{0}^{\infty} \beta(a, u) \rho(t, a) d a, \\
& \rho(0, a)=\rho_{0}(a) \geq 0, \quad u(t)=\int_{0}^{\infty} \rho(t, a) d a .
\end{aligned}
$$

1.2. Diffusion of populations. Let $\rho(\mathbf{x}, t)$ be the number of individuals present at time $t$ at position $\mathbf{x}, \mathbf{x} \in \mathbf{R}^{N}$. Immigration and emigration are modeled here by diffussion

$$
\rho_{t}+\operatorname{div} \mathbf{v}=\sigma
$$

where $\mathbf{v}(\mathbf{x}, t)$ is the diffusion velocity and $\sigma(\mathbf{x}, t)$ the population supply. The first model of this type was given by Skellam [28] in 1951. Assuming random motion of the individuals, $\mathbf{v}=-k \nabla \rho$, he arrived at

$$
\rho_{t}=k \Delta \rho+\sigma(t),
$$

where $k$ and $\mu$ are constants. It has been observed however that several species instead of dispersing at random actually disperse to avoid crowding (see for instance [7]). This corresponds to $\mathbf{v}=-k \rho \nabla \rho$ which gives

$$
\rho_{t}=k \operatorname{div}(\rho \nabla \rho)+\sigma .
$$

In [12] Gurney and Nibset arrived at this equation after considering a probabilistic walk in which individuals either stay at their present location or move in a direction of decreasing population. In [16] Gurtin and MacCamy considered

$$
\rho_{t}=\Delta \varphi(\rho)+\sigma(\rho)
$$

where $\varphi$ has properties similar to $\varphi(\rho)=\rho^{m}, m \geq 1$.

When $\sigma=0$ the equation

$$
\rho_{t}=\Delta\left(\rho^{m}\right)
$$

is the porous medium equation which models the flow of a homogeneous gas with density $\rho$ flowing through a homogeneous porous medium. There is an extensive literature for this equation (see for instance $[1,26,29]$ and the references contained there). The most striking difference between the solutions of (1.10) and those of the usual heat equation

$$
\rho_{t}=\Delta \rho
$$

is their speed of propagation: Assume the population is initially distributed in a bounded region $\Omega$ in space, i.e., $\operatorname{supp} \rho_{0} \subseteq \Omega$. The solutions of $(1.11)$ have an infinite speed of propagation: $\rho(\mathbf{x}, t)>0$ for $\mathbf{x} \in \mathbf{R}^{n}, t>0$, thus the population would spread immediately to all the space. On the other hand Eq. (1.10) has a finite speed of propagation. There are two fronts that separate the populated region $\rho(\mathbf{x}, t)>0$ from the unpopulated region $\rho(\mathbf{x}, t)=0$.

1.3. Age dependence and diffusion. In 1981 Gurtin and MacCamy $[14,17]$ proposed a complete model with age dependence and diffusion. They let $\rho(\mathbf{x}, t, a)$ be 
the population density at time $t$, age $a$ and spatial position $\mathbf{x}$. The anticrowding model is:

$$
\begin{gathered}
\rho_{t}+\rho_{a}=\kappa \operatorname{div}(\rho \nabla u)-\mu(a, u) \rho, \\
\rho(\mathbf{x}, t, 0)=\int_{0}^{\infty} \beta(a, u) \rho(\mathbf{x}, t, a) d a, \\
u(\mathbf{x}, t)=\int_{0}^{\infty} \rho(\mathbf{x}, t, a) d a, \\
\rho(\mathbf{x}, 0, a)=\rho_{0}(\mathbf{x}, a) \geq 0 .
\end{gathered}
$$

This system is too general to be treated in this form and some simplifying assumptions are necessary.

The model $(1.12)-(1.15)$ can be reduced to a system of partial differential equations when the birth modulus $\beta$ has the form $\beta(a, u)=\beta(u) e^{-\alpha a}$ and the death modulus $\mu$ depends on $u$ alone. These assumptions correspond to the case in which individuals are more fertile at younger age and age is not a significant cause of death. This is typically true both in the case of a population exposed to a harsh environment and that of a population in the presence of predators that do not discriminate by age. We introduce the auxiliary function $G(\mathbf{x}, t)=\int_{0}^{\infty} e^{-\alpha a} \rho(\mathbf{x}, t, a) d a$ and the per capita distribution term

$$
p(\mathbf{x}, t)=\frac{G(\mathbf{x}, t)}{u(\mathbf{x}, t)}
$$

for $u \neq 0$, and $p=0$ for $u=0$.

Integrating (1.12) with respect to $a$ from 0 to $\infty$ and multiplying by $e^{-\alpha a}$ and integrating with $\kappa$ normalized to be 1 , one arrives at the system

$$
\begin{aligned}
& u_{t}=u \Delta u+|\nabla u|^{2}+(\beta(u) p-\mu(u)) u, \\
& p_{t}-\nabla u \nabla p=(\beta(u)-\alpha) p-\beta(u) p^{2},
\end{aligned}
$$

and the initial conditions

$$
\begin{gathered}
u(\mathbf{x}, 0)=u_{0}(\mathbf{x})=\int_{0}^{\infty} \rho_{0}(\mathbf{x}) d \mathbf{x}, \\
p(\mathbf{x}, 0)=p_{0}(\mathbf{x})=\frac{\int_{0}^{\infty} e^{-\alpha a} \rho_{0}(\mathbf{x}, a) d a}{\int_{0}^{\infty} \rho_{0}(\mathbf{x}, a) d a} .
\end{gathered}
$$

This is a mixed system. The first equation is of porous medium type. It is nonlinear parabolic for $u(\mathbf{x}, t) \geq 0$, but it degenerates to $u_{t}=|\nabla u|^{2}$ at the points $u(\mathbf{x}, t)=0$. The second equation is of first order nonlinear hyperbolic type.

In [17] Gurtin and MacCamy treat the case in which $\beta(a, u)=\hat{\beta} e^{-\alpha a}, \hat{\beta}$ and $\mu$ constants, in the one-dimensional domain $0 \leq x \leq 1$, obtaining existence of a solution under these conditions.

Because of the similarity of (1.16) and (1.10) we should expect that the population $u(x, t)$ would disperse at a finite speed. Also under specific conditions on the birth and death modulus the population might remain localized for all times or under other assumptions extend to all of $\mathbf{R}^{N}$. In the one-dimensional case these results 
were proven in [20] and [19]. Namely, there exist two interfaces that separate the populated region from the unpopulated region, i.e., the support of $u(\mathbf{x}, t)$ is finite for all time. Also if

$$
\sup _{0 \leq u \leq M_{1}} \frac{\mu(u)}{\beta(u)}<\inf _{0 \leq u \leq M_{1}} \frac{\beta(u)-\alpha}{\beta(u)}
$$

then the support of $u$ grows to $\mathbf{R}$ as $t \rightarrow \infty$. In this case all the region will be ultimately populated. On the other hand if

$$
\sup _{0 \leq u \leq M_{1}} \frac{\beta(u)-\alpha}{\beta(u)}<\inf _{0 \leq u \leq M_{1}} \frac{\mu(u)}{\beta(u)}
$$

then the population remains localized in an interval $[-L, L]$ for all times. Here the interaction between age dependence and diffusion is such that the population persists in a limited region.

1.4. Weak solutions. It is well known $[23,3]$ that the porous medium equation (1.10) even with real analytic data will not have classical solutions unless $u_{0}$ is strictly positive in $\mathbf{R}^{N}$. This is due to the fact that if $u_{0}(x)$ has compact support the solutions will not have a continuous first derivative when crossing the interfaces. Thus we need to introduce a suitable definition of weak solutions of (1.16)-(1.19). Assume $u$ and $p$ are classical solutions. Multiplying (1.16) by $p,(1.17)$ by $u$ and adding we arrive at

$$
(u p)_{t}=\operatorname{div}\left(p \nabla u^{2}\right)+(\beta(u)-\alpha-\mu) u p .
$$

We define a test function $\varphi(\mathbf{x}, t)$ as a continuously differentiable function in $\Omega_{T}=\mathbf{R}^{N} \times(0, T)$ with compact support in $\bar{\Omega}_{T}=\mathbf{R} \times[0, T]$ and that equals 0 near $T$. Multiplying (1.16) and (1.20) by $\varphi(\mathbf{x}, t)$, integrating and using the divergence theorem we arrive at

$$
\begin{aligned}
& \iint_{\Omega_{T}}\left(\frac{1}{2} \nabla u^{2} \nabla \varphi-u \varphi_{t}\right) d \mathbf{x} d t \\
& \quad=\iint_{\Omega_{T}}(\beta(u) p-\mu(u)) u \varphi d \mathbf{x} d t+\int_{\mathbf{R}^{N}} u_{0}(\mathbf{x}) \varphi(\mathbf{x}, 0) d \mathbf{x}, \\
& \iint_{\Omega_{T}}\left(\frac{1}{2} p \nabla u^{2} \nabla \varphi-p u \varphi_{t}\right) d \mathbf{x} d t \\
& =\iint_{\Omega_{T}}(\beta(u)-\alpha-\mu(u)) p u \varphi d \mathbf{x} d t+\int_{\mathbf{R}^{N}} u_{0}(\mathbf{x}) p_{0}(\mathbf{x}) \varphi(\mathbf{x}, 0) d \mathbf{x} .
\end{aligned}
$$

We define a weak solution of (1.16)-(1.19) as a pair of functions $u(\mathbf{x}, t), p(\mathbf{x}, t)$ such that $u \in \mathscr{C}\left(\Omega_{T}\right), u^{2}$ has partial derivatives in the sense of distributions, $p \in$ $\mathscr{L}_{\text {loc }}^{2}\left(\Omega_{T}\right)$ and (1.21), (1.22) are satisfied for any test function $\varphi(\mathbf{x}, t)$.

The proof of existence of solutions of (1.16)-(1.19) was given in [20] for the one-dimensional case. There are no results in anticrowding model (1.16)-(1.19) for $N>1$. This is due to the fact that the porous medium equation (1.10) is much better understood in dimension 1 than in higher dimensions. 
In the one-dimensional case of (1.10) Aronson [2] proved that if $u_{0}^{m-1}(x)$ is Lipschitzian then $v(x, t)=u^{m-1}(x, t)$ is also Lipschitzian with respect to $x \in$ $\mathbf{R} \times(0, T)$. Benilam [4] and Aronson and Caffarelli [8] proved that $v$ also satisfies a Lipschitz condition with respect to $t$ in the same domain. In particular $u(x, t)$ is $\alpha$-Hölder continuous for any $\alpha \in(0,1)$, i.e., the $\alpha$-norm of $u$

$$
|u|_{\alpha}=\sup |u|+\sup \frac{|u(x, t)-u(y, \tau)|}{|x-y|^{\alpha}+|t-\tau|^{\alpha / 2}}
$$

is bounded by a constant $K$ that depends only on $u_{0}, m$, and $T$. In higher dimensions Caffarelli and Friedman [5] proved that $u(\mathbf{x}, t)$ is continuous with modulus of continuity

$$
w(\rho)=C|\log \rho|^{-\varepsilon}, \quad N \geq 3,0<\varepsilon<\frac{2}{N},
$$

and

$$
w(\rho)=2^{-c}|\log \rho|^{1 / 2}, \quad N=2,
$$

where $\rho=\left(|x-y|^{2}+|t-\tau|\right)^{1 / 2}$ is the parabolic distance between $(x, t)$ and $(y, \tau)$. Thus if $u_{0}$ is $\alpha$-Hölder continuous for some $\alpha \in(0,1)$ then $|u(x, t)-u(y, \tau)| \leq$ $w(\rho)$ uniformly in $\mathbf{R}^{N} \times[0, T]$. The same authors in [6] proved that $u(x, t)$ is actually $\alpha$-Hölder continuous for some $\alpha \in(0,1)$ but $\alpha$ is unknown. In [1] Aronson describes an example due to Graveleau which shows that if the support of $u_{0}$ has holes then it is possible for $\nabla u$ to blow up near the boundary. Hence $v(\mathbf{x}, t)$ cannot in general be Lipschitzian in $\mathbf{R}^{N} \times(\tau, T)$ for arbitrarily small $\tau$. Specifically for the porous medium problem in the radially symmetric case, if the gas lies initially completely outside a ball around 0 , as time increases the gas will fill the ball and ultimately reach its center. The Aronson-Graveleau example shows that at the moment $v(r, t)$ is like $r^{\alpha}$, where $\alpha \in(0,1)$ depends on the dimension $N$ and the constant $m$. For $N=1, \alpha(1, m)=1$. For $N>1, \alpha$ has only been estimated numerically. For example, it is given in [1] that $\alpha(2,2)=.832221204 \ldots$.

The one-dimensional porous medium type problem

$$
\begin{aligned}
u_{t} & =\left(u^{m}\right)_{x x}+h(x, t, u) u, \\
u(x, 0) & =u_{0}(x) \geq 0,
\end{aligned}
$$

was treated by the author in [21]. It is shown there that the corresponding $v(x, t)$ is $\alpha$-Hölder continuous for any $\alpha \in(0,1)$ provided $v_{0}$ is $\alpha$-Hölder continuous and $h$ is bounded. The proof of existence given in [20] is largely based in this fact. Related results are given by Di Benedetto [10], Paul Sacks [27], and others.

In this work we shall prove existence of weak solutions for radially symmetric initial distributions when $N \geq 3$. For the random dispersal model we refer the reader to Garroni-Langlais [11], Langlais [24], di Blasio [9] and the references contained there. 


\section{Main results.}

2.1. Statement of existence of solutions. Let $r=|\mathbf{x}|=\left(\sum_{i=1}^{N} x_{i}^{2}\right)^{1 / 2}$ be the Euclidean norm in $\mathbf{R}^{N}$. Radial solutions $u(r, t), p(r, t)$ of (1.16)-(1.19) satisfy

$$
\begin{gathered}
u_{t}=u u_{r r}+u_{r}^{2}+(N-1) \frac{u u_{r}}{r}+(\beta(u) p-\mu(u)) u, \\
p_{t}-u_{r} p_{r}=(\beta(u)-\alpha) p-\beta(u) p^{2}, \\
u_{r}(0, t)=0, \quad u(r, 0)=u_{0}(r), \quad p(r, 0)=p_{0}(r) .
\end{gathered}
$$

We shall assume that the population is initially concentrated in a ball $B\left(0, R_{1}\right)$ and it is strictly positive in some interior ball $B\left(0, R_{2}\right)$. From the definition of $u$ and $G$ it is easy to see that if $\rho_{0}(\mathbf{x}, a)=0$ a.e. with respect to $a$ then $u_{0}(\mathbf{x})=G_{0}(\mathbf{x})=0$. On the other hand if $\rho_{0}(\mathbf{x}, a)>0$ in a set of positive measure, then $0<G_{0}(\mathbf{x})<$ $u_{0}(\mathbf{x})$ and $p_{0}(\mathbf{x})<1$. The birth and death modulus $\beta$ and $\mu$ are assumed to be smooth and bounded along with their derivatives. We introduce a final change of dependent variable $q(r, t)=e^{\alpha t} p(r, t)$, and the problem to be considered is

$$
\begin{gathered}
u_{t}=u u_{r r}+u_{r}^{2}+(N-1) \frac{u u_{r}}{r}+\left(\beta(u) e^{-\alpha t} q-\mu(u)\right) u, \\
q_{t}-u_{r} q_{r}=\beta(u) q\left(1-e^{-\alpha t} q\right), \\
u_{r}(0, t)=0, \quad u(r, 0)=u_{0}(r), \quad q(r, 0)=q_{0}(r),
\end{gathered}
$$

where it is assumed $0<\bar{m} \leq q_{0}(r) \leq 1,0<\eta_{0} \leq u_{0}(r) \leq M_{0}$ on $\left[0, R_{2}\right) u_{0}(r) \equiv 0$ for $r>R_{1}$, and $\beta, \mu,\left|\beta^{\prime}\right|,\left|\mu^{\prime}\right| \leq M_{0}, \beta, \mu \geq 0$.

We let $\mathscr{C}^{\alpha}(\Omega)$ be the Banach space consisting of functions whose $\alpha$-norms (1.23) are bounded in $\Omega$. Similarly define the spaces $\mathscr{C}^{1+\alpha}\left(\Omega, \mathscr{C}^{2+\alpha}(\Omega)\right.$ with the corresponding norms $|u|_{1+\alpha}=|u|_{\alpha}+\left|u_{r}\right|_{\alpha}$ and $|u|_{2+\alpha}=|u|_{1+\alpha}+\left|u_{r}\right|_{1+\alpha}+\left|u_{t}\right|_{1+\alpha}$.

The corresponding weak solutions are given by:

Definition. A weak solution of (2.4)-(2.6) is a pair of bounded functions $u, q$ such that $u$ is continuous in $\Omega_{T}=(0, \infty) \times(0, T), u^{2}$ is differentiable in the sense of distributions, $r^{N-1}\left(u^{2}\right)_{r} \in \mathscr{L}_{\text {Loc }}, q \in \mathscr{L}_{\text {Loc }}^{2}$ and for any $\varphi(r, t)$ which is 0 near $T$ and for $r$ large, the following two equalities are satisfied

$$
\begin{aligned}
& \iint_{\Omega_{T}} r^{N-1}\left(\frac{1}{2}\left(u^{2}\right)_{r} \varphi_{r}-u \varphi_{t}\right) d r d t \\
& \quad=\iint_{\Omega_{T}} r^{N-1}\left(\beta(u) e^{-\alpha t} q-\mu(u)\right) u \varphi d r d t+\int_{0}^{\infty} r^{N-1} \varphi(r, 0) u_{0}(r) d r, \\
& \iint_{\Omega_{T}} r^{N-1}\left(\frac{1}{2} q\left(u^{2}\right)_{r} \varphi_{r}-q u \varphi_{t}\right) d r d t \\
& \quad=\iint_{\Omega_{T}} r^{N-1}(\beta(u)-\mu(u)) q u \varphi d r d t+\int_{0}^{\infty} r^{N-1} u_{0}(r) q_{0}(r) \varphi(r, 0) d r .
\end{aligned}
$$

Our first result establishes the existence of solutions for the radially symmetric Gurtin-MacCamy model.

THEOREM 1. Under the previous hypothesis there exists a weak solution for the system $(2.4)-(2.6)$. 
Proof. We shall follow the approach presented in [20] using a fixed point technique. The proof is long and it is done in several steps: First we prove existence of solutions of a smoother version of (2.4)-(2.6) depending on two parameters $\varepsilon$ and $n$. Then we let $n \rightarrow \infty$ and $\varepsilon \rightarrow 0$ after proving a priori bound for these solutions. Our main tool is an appropriate estimate for the Hölder modulus of continuity of radial solutions of $(2.4)$.

Let $y=(r, t)$. In $\mathbf{R}^{2}$ we consider a mollifier $J(y)$, a symmetric $\mathscr{C}^{\infty}$-function such that $J(y) \geq 0$ if $|y| \leq 1$ and $\int_{\mathbf{R}^{2}} J(y) d y=1$. (For instance

$$
J(y)= \begin{cases}k e^{-1 /\left(1-|y|^{2}\right)} & |y|<1, \\ 0 & |y|>1,\end{cases}
$$

for appropriate constant $k$.) We let $J_{n}(y)=\left(1 / n^{2}\right) J(n y)$. It is then clear that if $q \in \mathscr{L}^{2},\left\{q * J_{n}\right\}$ is a $\mathscr{C}^{\infty}$-sequence that converges to $q$ in $\mathscr{L}^{2}$, and if $q$ is continuous $\left\{q * J_{n}\right\}$ converges to $q$ uniformly on compact sets. We will apply Schauder's fixed point theorem to the following $\varepsilon$ - $n$-approximating systems:

$$
\begin{aligned}
u_{t}=u u_{r r} & +u_{r}^{2}+(N-1) \frac{(u-\varepsilon) u_{r}}{r+\varepsilon}+\left(\beta(u) e^{-\alpha t} z-\mu(u)\right)(u-\varepsilon), \\
q_{t} & -u_{r} q_{r}=\beta(u) q\left(1-e^{-\alpha t} q\right), \\
z & =\frac{1}{n^{2}} \int_{\left|y-y^{\prime}\right| \leq(1 / n)} J\left(n\left(y-y^{\prime}\right)\right) q\left(y^{\prime}\right) d y^{\prime}, \\
u_{r}(0, t) & =0, \quad u(r, 0)=u_{0}(r)+\varepsilon, \quad q(r, 0)=q_{0}(r) .
\end{aligned}
$$

Here $\varepsilon$ is introduced to regularize the porous medium part and $n$ is introduced to smooth out the term $h(r, t, u)=\left(\beta(u) e^{-\alpha t} z-\mu(u)\right)(u-\varepsilon)$ in $(2.4), z=q * J_{n}$.

2.2. Solution of the equation for $q$. We begin by studying the existence and regularity of solutions of $(2.10)$ for $u$ given. In this case the equation is nonlinear hyperbolic first order and can be solved by integration along characteristics.

Lemma 1. Assume $u \in \mathscr{C}^{2+\alpha}\left(\Omega_{T}\right)$. Then (2.10) has a unique solution $q \in$ $\mathscr{C}^{1+\alpha}\left(\Omega_{T}\right)$. This solution has $q(r, 0)=q_{0}(r)$ and $\bar{m} \leq q \leq e^{\alpha t}$.

Proof. We define characteristic curves $r(t ; x, \tau)$ by

$$
\begin{gathered}
\frac{\partial r}{\partial t}=-u_{r}(r(t), t), \\
r(\tau ; x, \tau)=x .
\end{gathered}
$$

Since $\bar{m} \leq q(r(0), 0) \leq e^{\alpha t}$, it follows that any solution $q$ increases along characteristics and $\bar{m} \leq q \leq e^{\alpha t}$. In particular we expect $q$ to be positive. Let $Q(r, t)=q^{-1}(r, t)$. Along characteristics we have:

$$
\begin{aligned}
\frac{\partial Q}{\partial t}+\beta(u) Q & =\beta(u) e^{-\alpha t}, \\
Q(r, 0) & =q_{0}^{-1}(r(0)) .
\end{aligned}
$$

Upon integration (2.15) yields

$$
q(x, \tau)=e^{\int_{0}^{\tau} \beta(u) d s}\left[q_{0}^{-1}(r(0))+\int_{0}^{\xi} \beta(u) e^{\int_{0}^{\sigma}(b(u)-\alpha) d \sigma} d \xi\right]^{-1} .
$$


Since $u_{r}$ is Lipschitzian there is always a local solution of (2.13)-(2.14). Since $u_{r}$ is bounded this solution can be extended to the boundary of $\Omega_{T}$.

Direct differentiation of $(2.16)$ shows that $q(x, \tau)$ is a solution of $(2.10),(2.12)$. Haar's lemma implies uniqueness. Since $u \in \mathscr{C}^{2+\alpha}\left(\Omega_{T}\right)$ then $q \in \mathscr{C}^{1+\alpha}\left(\Omega_{T}\right)$.

LemMA 2. There exists a solution of (2.9)-(2.12).

Proof. Let $K_{1}$ be a constant and denote $V=\left\{w \in \mathscr{C}^{2+\alpha}\left(\Omega_{T}\right) /|w|_{2+\alpha} \leq k_{1}\right.$, $w \geq \varepsilon\}$. Define $T: V \mapsto \mathscr{C}^{2+\alpha}\left(\Omega_{T}\right)$ in the following way: Given $w \in V$, by the previous lemma there exists a unique solution $q(w) \in \mathscr{C}^{1+\alpha}\left(\Omega_{T}\right)$ and $\bar{m} \leq q \leq e^{\alpha t}$. Then $z \in \mathscr{C}^{\infty}\left(\Omega_{T}\right)$ and $|z|_{\sigma} \leq 2 n$ for any $\sigma \in(0,1)$, so by standard results in parabolic differential equations coupled with the fact that $u(r, 0) \geq \varepsilon$, there exists a unique solution $u \in \mathscr{C}^{2+\sigma}\left(\Omega_{T}\right)$ with $|u|_{2+\sigma} \leq k_{2}$, where $k_{2}$ depends only on $n$ and $\varepsilon$; let $u=T(w)$.

Taking $k_{1} \leq k_{2}$ and $\sigma<\alpha$ we have that $T$ maps $V$ into $V$. Further, since bounded sets in $\mathscr{C}^{2+\sigma}\left(\Omega_{T}\right)$ are precompact in $\mathscr{C}^{2+\alpha}$ for $\sigma<\alpha$ we also have that $T(V)$ is precompact. It is clear that $T$ is continuous since the equations and functions involved are smooth (depending on $n$ and $\varepsilon$ ). It follows then by Schauder's fixed point theorem that $T$ has a fixed point $u=T(u)$. This $u$ and the corresponding $q$ and $z$ form a solution of (2.9)-(2.12).

Next we show that after letting $n$ tends to infinity we obtain a solution of the following $\varepsilon$-approximation problems:

$$
\begin{gathered}
u_{t}=u u_{r r}+(N-1) \frac{(u-\varepsilon) u_{r}}{r+\varepsilon}+\left(\beta(u) e^{-\alpha t} q-\mu(u)\right)(u-\varepsilon), \\
q_{t}-u_{r} q_{r}=\beta(u) q\left(1-e^{-\alpha t} q\right), \\
u_{r}(0, t)=0, \quad u(r, 0)=u_{0}(r)+\varepsilon, \quad q(r, 0)=q_{0}(r) .
\end{gathered}
$$

2.3. Estimates for $u$. The solutions of (2.9) with the initial conditions in (2.12) can be obtained as limits when $R \rightarrow \infty$ of the solution of the problems

$$
\begin{gathered}
u_{t}=E(u) u_{r r}+u_{r}^{2}+(N-1) \frac{(u-\varepsilon) u_{r}}{r+\varepsilon} \\
+\left(\beta(u) e^{-\alpha t} z-\mu(u)\right)(u-\varepsilon), \\
u(r, 0)=u_{0}(r)+\varepsilon, \quad u_{r}(0, t)=0, \quad u(R, t)=\varepsilon,
\end{gathered}
$$

where $E(z)$ is a $\mathscr{b}^{\infty}$-function satisfying $E(z)=z$ for $z \geq \varepsilon, E(z)=\frac{\varepsilon}{2}$ for $z \leq \frac{\varepsilon}{2}$ and $E(z)$ increases from $\frac{\varepsilon}{2}$ to $\varepsilon$ in $\frac{\varepsilon}{2} \leq z \leq \varepsilon$.

The function $v=e^{-M_{0} t}(u-\varepsilon)$ satisfies

$$
\begin{gathered}
v_{t}=E(u) v_{r r}+e^{M_{0} t} v_{r}^{2}+(N-1) e^{M_{0} t} \frac{v v_{r}}{r+\varepsilon}+\left(h-M_{0}\right) v, \\
v(r, 0)=u_{0}(r), \quad v_{r}(0, t)=0, \quad u(R, t)=0 .
\end{gathered}
$$

Since $h \leq M_{0}$, the maximum principle implies that $0 \leq v(r, t) \leq M_{0}$, independently of $R$. Therefore $\varepsilon \leq u(r, t) \leq M_{1}=M_{0} e^{M_{0} T}$ in $\Omega_{T}$.

Now we derive an appropriate estimate for the gradient of $u$ and the gradient of $q$. 
Lemma 3. Let $u \in \mathscr{C}^{2+\alpha}\left(\Omega_{T}\right)$ be a solution of (2.9), $z \in \mathscr{C}^{1+\alpha}\left(\Omega_{T}\right)$. Then there exist constants $K_{1}, K_{2}$ (depending on $\varepsilon$ ) such that for any $c_{1}>0$

$$
\left|u_{r}\right|_{\infty}^{2} \leq K_{1}+\frac{K_{2}}{c_{1}}+c_{1}\left|z_{r}\right|_{\infty}^{2} \text {. }
$$

Proof. This is an application of Bershtein's technique as in Oleinik [25] and Aronson [2]. The details involve some straightforward calculations that are only sketched.

Let $\varphi(y)=\left(M_{1} / 3\right) y(4-y) . \varphi:[0,1] \mapsto\left[0, M_{1}\right]$ with positive first derivatives bounded away from 0 and negative second derivative. Let $w=\varphi^{-1}(u)$, then $w$ satisfies

$$
\begin{aligned}
w_{t}= & \varphi w_{r r}+\left[\varphi^{\prime}+\frac{\varphi \varphi^{\prime \prime}}{\varphi^{\prime}}+\frac{N-1}{r+\varepsilon} \varphi\right] w_{r} \\
& +\left(\beta(\varphi) e^{-\alpha t} z-\mu\right)\left(\frac{\varphi-\varepsilon}{\varphi}\right) .
\end{aligned}
$$

Differentiating with respect to $r$ and letting $v=w_{r}$, we obtain

$$
\begin{aligned}
\frac{1}{2}\left(v_{t}^{2}-\varphi v v_{r r}\right)= & A v^{2} v_{r}+\frac{N-1}{r+\varepsilon} \varphi^{\prime} v^{3}+B v^{4}+\frac{N-1}{r+\varepsilon}(\varphi-\varepsilon) v v_{r} \\
& -\frac{N-1}{(r+\varepsilon)^{2}}(\varphi-\varepsilon) v^{2}+C v^{2}+D v z_{r},
\end{aligned}
$$

where the coefficients $A, B, C, D$ depend only on $\varphi, \varphi^{\prime}, \varphi^{\prime \prime}$. Next take a cutoff function $\xi(r) \in \mathscr{C}^{\infty}(\mathbf{R}), \xi(r)=0$ for $r \geq m_{1}+1$ and $\xi(r) \equiv 1$ for $0 \leq r \leq m_{1}$ and let $p=\xi^{2} v^{2}$. At an interior maximum of $p$ we should have

$$
p_{r}=0, \quad p_{t}-\varphi p_{r r} \geq 0 .
$$

Using (2.23) and (2.25) we arrive at

$$
\begin{aligned}
-2 \xi^{2} B v^{4} \leq & {\left[2 \frac{N-1}{r+\varepsilon} \varphi^{\prime} \xi-2 A \xi_{r}\right] \xi v^{3} } \\
& +\left[2 \frac{N-1}{r+\varepsilon}(\varphi-\varepsilon) \xi \xi_{r}-2 \frac{N-1}{(r+\varepsilon)^{2}}(\varphi-\varepsilon) \xi^{2}\right] v^{2}+2 D \xi^{2}\left|z_{r}\right| v .
\end{aligned}
$$

Let $E$ and $F$ be the coefficients of $v^{3}$ and $v^{2}$, respectively. Note that

$$
-B=-\left(\varphi^{\prime}+\frac{\varphi \varphi^{\prime \prime}}{\varphi^{\prime}}\right)^{\prime} \geq \frac{3}{2} M_{1}
$$

and the triangle inequality gives

$$
\xi v^{3} \leq \frac{v^{2} E^{2}}{4 M}+M \xi^{2} v^{4}
$$

thus for any $c_{2}>0$

$$
2|D| \xi^{2} v^{4} \leq\left(\frac{E^{2}}{4 m}+F+\frac{D^{2}}{c_{2}^{2}}\right) v^{2}+c_{2}^{2}\left|z_{r}\right|_{\infty}^{2} .
$$


From here it follows that there exist constants $K_{1}^{\prime}, K_{2}^{\prime}$ such that

$$
\xi^{2} v^{2} \leq K_{1}^{\prime}+\frac{K_{2}^{\prime}}{c_{2}^{2}}+c_{2}^{2}\left|z_{r}\right|_{\infty}^{2}
$$

for $c_{2}>0$ arbitrary.

Since

$$
u_{r}^{2}=\varphi^{\prime 2} w_{r}^{2}=\varphi^{\prime 2} \xi^{2}(r) w_{r}^{2}
$$

for $r \in\left(0, m_{1}\right)$ we have

$$
u_{r}^{2} \leq K_{1} \varphi^{\prime 2}+\frac{K_{2}^{\prime} \varphi^{\prime 2}}{c_{2}^{2}}+c_{2}^{2} \varphi^{\prime 2}\left|z_{r}\right|_{\infty}^{2} .
$$

Since $m_{1}$ is arbitrary and $\varphi^{\prime} \leq\left(2 M_{1} / 3\right)$ this proves the lemma.

2.4. Estimates for $q$.

LemMA 4. Let $q \in \mathscr{C}^{1+\alpha}\left(\Omega_{T}\right)$ be a solution of (2.10) where $u$ is a solution of (2.9). Then there exist constants $K_{3}, K_{4}$ (depending on $\varepsilon$ ) such that

$$
\left|q_{r}\right| \leq K_{3}\left|u_{r}\right|_{\infty} \text { and }\left|q_{t}\right| \leq K_{4}\left|u_{r}\right|_{\infty}^{2} .
$$

Proof. Differentiating with respect to the parameter $x$ in (2.15) we obtain

$$
\begin{aligned}
\frac{\partial r_{x}}{\partial t} & =-u_{r r} r_{x}, \\
r_{x}(\tau) & =1 .
\end{aligned}
$$

Thus

$$
\begin{aligned}
r_{x} & =e^{\int_{\tau}^{t} u_{r r}(r(s), s) d s} \\
& \leq e^{\int_{\tau}^{t}\left(u_{t}-u_{r}^{2}\right) / u-\left[(N-1)\left(u_{r} /(r+\varepsilon)\right)-\left(\beta e^{-\alpha t} z-\mu\right)\right]((u-\varepsilon) / u) d s} \\
& \leq \frac{u(r(t), t)}{u(r(\tau), \tau)}+\left(\frac{r(t)+\varepsilon}{r(\tau)+\varepsilon}\right)^{N-1}+e^{|h| T} \\
& \leq K_{5}(\varepsilon) .
\end{aligned}
$$

The function $V(t)=r_{\tau}(t)-u_{r}(r, \tau) r_{x}(\tau)$ satisfies $V^{\prime}(t)=u_{r r} V(t)$ and $V(\tau)=0$. Thus $V \equiv 0$ and $r_{\tau}=u_{r}(r, x) r_{x}$. It follows that $r_{\tau} \leq K_{5}\left|u_{r}\right|_{\infty}$.

Differentiating expression (2.16) we have that $q_{x}$ and $q_{\tau}$ are bounded by multiples of $\beta^{\prime}, u_{r}, r_{x}, q_{0}^{-1}$, and $q_{0}^{\prime}$, and using the previous estimates we obtain that $\left|q_{x}\right|_{\infty}$ and $\left|q_{\tau}\right|_{\infty}$ are bounded by multiples of $\left|u_{r}\right|_{\infty}$ and $\left|u_{r}\right|_{\infty}^{2}$, respectively.

With the aid of the previous two lemmas we obtain bounds for $u_{r}^{n}$ and $q_{r}^{n}$ independently of $n$, for a solution $u^{n}, q^{n}$ of (2.9)-(2.12). By Lemma 4 we have $\left|q_{r}^{n}\right|_{\infty} \leq K_{3}\left|u_{r}^{n}\right|_{\infty}$, thus $\left|z_{r}^{n}\right|_{\infty} \leq K_{3}\left|u_{r}^{n}\right|_{\infty}$ and by Lemma 3, with $c_{1}=1 / 2 K_{3}^{2}$ we have

$$
\left|u_{r}^{n}\right|_{\infty}^{2} \leq K_{1}+2 K_{2} K_{3}^{2}+\frac{1}{2}\left|u_{r}^{n}\right|_{\infty}^{2},
$$

i.e.,

independently of $n$.

$$
\left|u_{r}^{n}\right|_{\infty}^{2} \leq 2\left(K_{1}+2 K_{2} K_{1}^{2}\right)
$$


Also

$$
\left|q_{r}^{n}\right|_{\infty} \leq K_{3}\left(2\left(K_{1}+2 K_{2} K_{1}^{2}\right)\right)^{1 / 2}
$$

is uniformly bounded.

Since $\left|q^{n}\right|_{\alpha}$ and $\left|z^{n}\right|_{\alpha}$ are bounded independently of $n$ we can extract a subsequence $\left\{q^{n_{k}}\right\}$ of $\left\{q^{n}\right\}$ that converges uniformly to a function $q_{\varepsilon}$ on compact subsets of $\Omega_{T}$. The corresponding sequence $\left\{z^{n_{k}}\right\}$ converges uniformly to $q_{\varepsilon}$. We rename these sequences $\left\{q^{n}\right\}$ and $\left\{z^{n}\right\}$. Since $u^{n} \geq \varepsilon$, Eqs. (2.9) are uniformly parabolic in $n$. It follows then from the standard theory that $\left|u^{n}\right|_{2+\alpha} \leq K_{6}$, a constant independent of $n$, and for $\alpha^{\prime}<\alpha$ there exists a subsequence $\left\{u^{n_{k}}\right\}$ that converges to a function $u_{\varepsilon}$ in $\mathscr{C}^{2+\alpha^{\prime}}\left(\Omega_{T}\right)$. In particular $\left\{u^{n_{k}}\right\}$ and $\left\{u_{r}^{n_{k}}\right\}$ converge uniformly in compact sets to $u_{\varepsilon}$ and $u_{r}^{\varepsilon}$, respectively.

The uniform boundedness of $\left|u_{r}^{n_{k}}\right|_{\alpha}$ implies that also $q_{\varepsilon}$ satisfies (2.16), thus the pair $u_{\varepsilon}, q_{\varepsilon}$ is a solution of (2.17)-(2.19).

2.5. The $\alpha$-norm of $u$. Next it is shown that $\left\{u_{\varepsilon}\right\},\left\{q_{\varepsilon}\right\}$ converge to a weak solution of $(2.4)-(2.6)$. We start with the following fundamental result which is important in its own right.

TheOREM 2. The $\alpha$-norms of the solutions $\left\{u_{\varepsilon}\right\}$ are uniformly bounded in $\Omega_{T}$ for $0<\alpha<1$, i.e., there exists $K^{*}>0$ such that

$$
\frac{|u(x, t)-u(y, \tau)|}{|x-y|^{\alpha}+|t-\tau|^{\alpha / 2}} \leq K^{*},
$$

and $K^{*}$ depends only on the data $M_{0}, \alpha$, and $T$.

Proof. A more general result with $u_{0}(r)=0$ in a neighborhood of 0 has been proved in [22]. Here, the fact that $u_{0}>0$ on $\left[0, R_{2}\right]$ will simplify the proof and also will allow us to obtain bounded $\alpha$-norm of any $\alpha \in(0,1)$. In the general case treated in [22] one only gets the $\alpha$-norm bounded for $\alpha \leq 1 / 2(N-2), N \geq 3$.

Let $h(r, t, u)=\beta(u) e^{-\alpha t} q-\mu(u), u=u_{\varepsilon}$. Then (2.9) is

$$
\begin{gathered}
u_{t}=u u_{r r}+u_{r}^{2}+(N-1) \frac{(u-\varepsilon) u_{r}}{r+\varepsilon}+h(r, t, u)(u-\varepsilon), \\
u_{r}(0, t)=0, \quad u(r, 0)=u_{0}(r)+\varepsilon .
\end{gathered}
$$

We begin with the Hölder continuity with respect to $r$. Since $u$ is a classical solution in $\Omega_{T}$ we can choose $\delta$ small such that $\left|u_{t}\right|_{\infty},\left|u_{r}\right|_{\infty} \leq \delta^{\alpha-1}$ in $\Omega_{T}$ and $\delta \leq r_{0}=R_{2} / 2$.

Lemma 5. Let $S_{\delta, R}=\left\{(r, s) \in \mathbf{R}^{2}: \delta<s<s+\delta<r<R\right\}, B_{\delta, R}=S_{\delta} \times(0, T)$ and define for $\alpha \in(0,1)$ fixed

$$
g(r, s, t)=\frac{|u(r, t)-u(s, t)|^{\lambda}}{(r-s)^{2}}
$$

$\lambda=\frac{2}{\alpha}$. Then $g$ is bounded in $B_{\delta}$ (indendently of $\delta$ and $R$ ).

Proof. Clearly $g$ is continuous, so it must attain its maximum at a point $Q_{1}=$ $\left(r_{1}, s_{1}, t_{1}\right) \in B_{\delta}$. Either $Q_{1}$ is an interior point at which $g$ is differentiable or $Q_{1}$ 
is a point on the boundary of $B_{\delta, R}$ ( $g$ is not differentiable only at those points $(r, t, s)$ where $g(r, t, s)=0$. We begin with the former possibility.

In this case we must have

$$
g_{r}=g_{s}=0, \quad g_{r r}, g_{s s} \leq 0, \quad g_{t} \geq 0,
$$

and

$$
E=v_{1} g_{r r}+v_{2} g_{s s}-g_{t} \leq 0 \text { at } Q_{1},
$$

where $v_{1}=v(r, t), v_{2}=v(s, t)$. Let $S=\left|v_{1}-v_{2}\right|, \sigma=\operatorname{sgn}(S)$. The first derivatives are

$$
\begin{aligned}
& g_{r}=\sigma \lambda|S|^{\lambda-1} v_{1 r} R^{-2}-2|S|^{\lambda} R^{-3}, \\
& g_{s}=-\sigma \lambda|S|^{\lambda-1} v_{2 s} R^{-2}+2|S|^{\lambda} R^{-3}, \\
& g_{t}=\sigma \lambda|S|^{\lambda-1}\left(v_{1 t}-v_{2 t}\right) R^{-2} .
\end{aligned}
$$

Thus (2.31) implies

$$
v_{1 r}=\frac{2 \sigma}{\lambda}|S| R^{-1}=v_{2 s}
$$

and

$$
\begin{aligned}
& g_{r r}=2|S|^{\lambda} R^{-4}(1-\alpha)+\lambda|S|^{\lambda-1} \sigma R^{-2} v_{1 r r}, \\
& g_{s s}=2|S|^{\lambda} R^{-4}(1-\alpha)-\lambda|S|^{\lambda-1} \sigma R^{-2} v_{2 s s} .
\end{aligned}
$$

Replacing in $E$ we obtain

$$
2|S|^{\lambda} R^{-4}(1-\alpha)\left(v_{1}+v_{2}\right)+\lambda \sigma|S|^{\lambda-1} R^{-2}\left[\left(v_{1} v_{1 r r}-v_{1 t}\right)-\left(v_{2} v_{2 s s}-v_{2 t}\right)\right] \leq 0 .
$$

Using the differential equation (2.30) in $(r, t)$ and $(s, t)$ in the last term we have

$$
\begin{aligned}
2(1-\alpha)|S|^{\lambda} R^{-4}\left(v_{1}+v_{2}\right) & \\
+\lambda \sigma|S|^{\lambda-1} R^{-2}[ & -v_{1 r}^{2}-(N-1) \frac{\left(v_{1}-\varepsilon\right) v_{1 r}}{r+\varepsilon}-h\left(r, t, v_{1}\right)\left(v_{1}-\varepsilon\right) \\
& \left.+v_{2 s}^{2}+(N-1) \frac{\left(v_{2}-\varepsilon\right) v_{2 s}}{s+\varepsilon}+h\left(s, t, v_{2}\right)\left(v_{2}-\varepsilon\right)\right] \leq 0
\end{aligned}
$$

and using (2.32) with $h_{1}=h\left(r, t, v_{1}\right), h_{2}=h\left(s, t, v_{2}\right)$

$$
\begin{aligned}
& 2 \frac{|S|^{\lambda}}{R^{2}}\left[(1-\alpha)\left(v_{1}+v_{2}\right)+(N-1)(r-s)\left(\frac{\left(v_{2}-\varepsilon\right)}{s+\varepsilon}-\frac{\left(v_{1}-\varepsilon\right)}{r+\varepsilon}\right)\right] \\
& \quad+\lambda \sigma|S|^{\lambda-1}\left(h_{2}\left(v_{2}-\varepsilon\right)-h_{1}\left(v_{1}-\varepsilon\right)\right) \leq 0 .
\end{aligned}
$$

We drop the positive term in $\left(v_{2}-\varepsilon\right) /(s+\varepsilon)$. Since $|S| \leq 2 M_{1}$ we obtain,

$$
\begin{gathered}
2 \frac{|S|^{\lambda}}{R^{2}}\left[(1-\alpha)\left(v_{1}+v_{2}\right)-(N-1)(r-s) \frac{v_{1}-\varepsilon}{r+\varepsilon}\right] \\
\leq \lambda\left(2 M_{1}\right)^{\lambda-1}\left(h_{2}\left(v_{2}-\varepsilon\right)-h_{1}\left(v_{1}-\varepsilon\right)\right) .
\end{gathered}
$$


Let $\hat{\delta}=\left((1-\alpha) r_{0}\right) /(2(N-1))$. If $r-s<\hat{\delta}$ the coefficient on the left of $(2.36)$ is bounded below by $\frac{1}{2}(1-\alpha)\left(v_{1}+v_{2}\right)$ and

$$
\begin{aligned}
g_{\max } & =\frac{|S|^{\lambda}}{R^{2}} \leq \frac{\lambda}{2} \frac{\left(2 M_{1}\right)^{\lambda-1}}{1-\alpha}\left|\frac{h_{2}\left(v_{2}-\varepsilon\right)}{v_{1}+v_{2}}-\frac{h_{1}\left(v_{1}-\varepsilon\right)}{v_{1}+v_{2}}\right| \\
& \leq \frac{\left(2 M_{1}\right)^{\lambda-1}}{\alpha(1-\alpha)}\left(2 M_{0}\right) .
\end{aligned}
$$

On the other hand if $r-s>\hat{\delta}$, then directly from the definition of $g$

$$
g_{\max } \leq \frac{\left(2 M_{1}\right)^{\lambda-1}}{\hat{\delta}^{2}}<\frac{4(N-1)^{2}\left(2 M_{1}\right)^{\lambda-1}}{(1-\alpha)^{2} r_{0}^{2}} .
$$

A bound for the $\alpha$-Hölder quotient of $u$ at interior points of $B_{\delta}$ has thus been obtained.

If $Q_{1}$ lies on the boundary of $B_{\delta}$ we are led to consider four possibilities: (i) $Q_{1}$ lies on the interior boundary $r-s=\delta$, (ii) $Q_{1}$ lies on the interior boundary $s=\delta$, (iii) $Q_{1}$ lies on the lower boundary $t=0$, and (iv) $Q_{1}$ lies on the lateral boundary $r=R$. We treat each of these cases separately.

(i) If $r=s+\delta$ by the mean value theorem and the assumption that $\left|u_{r}\right|_{\infty} \leq \delta^{\alpha-1}$ we get

$$
g\left(Q_{1}\right) \leq \frac{\left|u_{r}(\cdot, t)\right|^{\lambda} \delta^{\lambda}}{\delta^{2}} \leq \delta^{\alpha-1} \delta^{\lambda-2}=1 .
$$

(ii) If $s=\delta$ by the standard regularity results for the porous medium equation with positive data there is a constant $K_{4}$ (independent of $\left.\delta, \varepsilon\right)$ such that $\left|u_{t}\right|_{\infty}$, $\left|u_{r}\right|_{\infty} \leq K_{4}$ on $\left[0, R_{2}\right]$. Thus, if $|r-s| \leq R_{2} / 2$, again by the mean value theorem

$$
g\left(Q_{1}\right)=\frac{\left|u_{2}-u_{1}\right|^{\lambda}}{(r-s)^{2}} \leq K_{4}^{\lambda}(r-s)^{\lambda-2} \leq K_{4}^{\lambda}\left(\frac{R_{2}}{2}\right)^{\lambda-2} .
$$

On the other hand if $|r-s| \geq R_{2} / 2$ then

$$
g\left(Q_{1}\right) \leq \frac{(2 M)^{\lambda}}{\left(R_{2} / 2\right)^{2}}
$$

(iii) If the maximum occurs at $t=0$ we use the initial data to get that

$$
g\left(Q_{1}\right) \leq M_{0} \text { when }|r-s| \leq 1
$$

and

$$
g\left(Q_{1}\right) \leq\left(2 M_{0}\right)^{\lambda} \quad \text { when }|r-s| \geq 1 .
$$

(iv) The case $r=R$ needs special consideration. We start by proving that the oscillation of $u$ tends to 0 as $r$ tends to infinity.

Lemma 6. Let $u$ be a solution of (2.30). Then for any $\eta>0$ there exists $R_{\eta}$ such that $|u(r, t)-u(s, t)| \leq \eta$ for $r, s \geq R_{\eta}, t \in[0, T]$.

Proof. We choose $R$ large such that $(N-1) u_{r} /(r+\varepsilon) \leq M_{0}$ and $u_{0}(r) \equiv 0$ for $r>R$. Write $(2.30)$ as

$$
u_{t}=u u_{r r}+u_{r}^{2}+\hat{h}(u-\varepsilon)
$$


where

$$
|\hat{h}(u, r, t)|=\left|(N-1) \frac{u_{r}}{r+\varepsilon}+\beta(u) q-\mu(u)\right| \leq 2 M_{0} .
$$

Without loss of generality we assume $M_{0} \geq 1$.

Let $t_{2}=1 /\left(24 M_{0}\right), \sigma<1$, and $m=\frac{1}{\sigma}$. Let $g(t)=2 t_{2} /\left(2 t_{2}-t\right)$ and consider

$$
v(r, t)=\sigma g(t)\left(r-r_{1}\right)^{2}+\varepsilon g^{\sigma}(t)
$$

on $B_{1}=\left[r_{1}-m, r_{1}+m\right] \times\left[0, t_{2}\right]$ and $r_{1}$ is such that $r_{1}-m \geq R$. We will use $v$ as an upper bound for $u$ and obtain $u(r, t) \leq 2^{\sigma} \varepsilon$ on $B_{1}$.

Let $\mathscr{L}[z]=z_{t}-z z_{r r}-z_{r}^{2}-\hat{h}(z, r, t)(z-\varepsilon)$. Then $\mathscr{L}[u]=0$ and

$$
\begin{aligned}
\mathscr{L}[v]= & \sigma \frac{\left(r-r_{1}\right)^{2} g^{2}(t)}{2 t_{2}}\left(1-12 \sigma t_{2}-\left(2 t_{2}-t\right) \hat{h}\right) \\
& +\varepsilon\left(\sigma \frac{g^{\sigma+1}(t)}{2 t_{2}}-2 a g^{\sigma+1}(t)-\hat{h}(u, r, t)\left(g^{\sigma}(t)-1\right)\right) .
\end{aligned}
$$

By the mean value theorem

$$
g^{\sigma}(t)-1=\sigma g^{\sigma-1}(s) g^{\prime}(s) t=\frac{\sigma t_{2}}{2 t_{2}} g^{\sigma+1}(s) \text { for some } s \in(0, t) .
$$

Since $|\hat{h}| \leq 2 M_{0}, 0 \leq t \leq t_{2}$, and $g(s) / g(t)=\left(2 t_{2}-t\right) /\left(2 t_{2}-s\right)$ lies between $\frac{1}{2}$ and 1 we have

$$
\begin{aligned}
\mathscr{L}[v] \geq & \sigma \frac{\left(r-r_{1}\right)^{2} g(t)^{2}}{2 t_{2}}\left(1-12 \sigma t_{2}-4 t_{2} M_{0}\right) \\
& +\varepsilon \sigma \frac{g(t)^{\sigma+1}}{2 t_{2}}\left(1-4 t_{2}-2 M_{0} t_{2}\right) \\
\geq & 0
\end{aligned}
$$

by the choice of $t_{2}$ and $\sigma$.

Next

$$
v(r, 0)=\sigma\left(r-r_{1}\right)^{2}+\varepsilon \geq \varepsilon=u(r, 0) \text { on }\left[r_{2}-m, r_{2}+m\right]
$$

and

$$
v\left(r_{2} \pm m, t\right)=\sigma \frac{2 t_{2} m^{2}}{2 t_{2}-t}+\varepsilon g^{\sigma}(t) \geq \sigma m^{2}=m
$$

Thus $v\left(r_{2} \pm m, t\right) \geq u\left(r_{2} \pm m, t\right)$ for $m \geq M_{1}$. It follows then by the maximum principle that $u \leq v$ on $\left[r_{1}-m, r_{1}+m\right]$. In particular

$$
u\left(r_{1}, t\right) \leq \varepsilon\left(\frac{2 t_{2}}{2 t_{2}-t}\right)^{\sigma} \leq 2^{1 / m} \varepsilon .
$$

Since the only restriction for $r_{1}$ is to be larger than $R+m$, Eq. (2.40) is valid for any $r \geq r_{1}$. We repeat the argument with $r_{2} \geq r_{1}+m$ on $\left[t_{2}, 2 t_{2}\right]$ with initial datum $u\left(r, t_{2}\right) \leq 2^{1 / m} \varepsilon$ and obtain that $u(r, t) \leq 2^{2 / m} \varepsilon$ for $r \geq r_{2} \geq R+2 m$. After $k=\left[T /\left(t_{2}+1\right)\right]$ steps we arrive at

$$
u(r, t) \leq 2^{k / m} \varepsilon \text { for } r \geq r_{k} \geq R+k m, \quad 0 \leq t \leq T .
$$


Now, given $\eta>0$, choose $m$ so large that also $2^{k / m} \leq 1+\eta / \varepsilon$. Then for $R_{\eta}=r_{k}$ we have $u(r, t) \leq \varepsilon+\eta$ for all $r \geq R_{\eta}, t \in[0, T]$. This proves the lemma.

We use this result in the proof of (iv). Choose $R$ so large so as to have $u(r, t) \leq$ $\varepsilon+\delta$ for $r \geq R-1$. Then if $|r-s| \geq 1$ we have

$$
g\left(Q_{1}\right) \leq\left(2 M_{1}\right)^{\lambda}
$$

and if $|r-s| \leq 1$ we have

$$
g\left(Q_{1}\right)=\frac{\left|u_{1}-u_{2}\right|^{\lambda}}{(r-s)^{2}} \leq \frac{\delta^{\lambda}}{\delta^{2}} \leq 1 .
$$

Therefore $g(r, s, t)$ is bounded in $B_{\delta}$ independently of $\delta$ and $\varepsilon$. Letting $\delta \rightarrow 0$ and $R \rightarrow \infty$, we obtain that $g(r, s, t)$ is bounded in $\Omega_{T}$. Lemma 6 implies that $g(r, s, t)^{\alpha / 2}$, the $\alpha$-quotient of $u$, is bounded in $\Omega_{T}$.

The result for $t$ follows in a similar way by considering the function

$$
k(r, s, t, \tau)=\frac{|u(r, t)-u(s, \tau)|^{\lambda}}{(r-s)^{2}+A|t-\tau|}
$$

at a point of maximum (we omit the details). This proves Theorem 2

2.6. Convergence of $\left\{u_{\varepsilon}\right\}$. Since $\left\{u_{\varepsilon}\right\},\left\{q_{\varepsilon}\right\}$ satisfy (2.17)-(2.19) we also have

$$
\begin{aligned}
\left(q_{\varepsilon} u_{\varepsilon}\right)_{t}= & \frac{1}{2}\left(q_{\varepsilon}\left(\left(u_{\varepsilon}\right)^{2}\right)_{r}\right)_{r}+\frac{1}{2} \frac{N-1}{r+\varepsilon} q_{\varepsilon}\left(\left(u_{\varepsilon}\right)^{2}\right)_{r} \\
& +\frac{1}{2} \frac{N-1}{r+\varepsilon} q_{\varepsilon}\left(\left(\left(u_{\varepsilon}-\varepsilon\right)^{2}\right)_{r}-\left(\left(u_{\varepsilon}^{2}\right)_{r}\right)\right) \\
& +\left[\beta\left(u_{\varepsilon}\right)-\mu\left(u_{\varepsilon}\right)\right] q_{\varepsilon} u_{\varepsilon}+\varepsilon q_{\varepsilon}\left[\mu\left(u_{\varepsilon}\right)-\beta\left(u_{\varepsilon}\right) e^{-\alpha t} q_{\varepsilon}\right] .
\end{aligned}
$$

Multiplying (2.17), (2.41) by $(r+\varepsilon)^{N-1}, \varphi(r, t)$ and integrating over $\Omega_{T}$ we obtain

$$
\begin{aligned}
\left.\iint_{\Omega_{T}}(r+\varepsilon)^{N-1}\left[\frac{1}{2}\left(\left(u_{\varepsilon}\right)^{2}\right)_{r} \varphi_{r}-u_{\varepsilon} \varphi_{t}\right)\right] d r d t \\
=\iint_{\Omega_{T}}(r+\varepsilon)^{N-1}\left[\beta\left(u_{\varepsilon}\right) e^{-\alpha t} q_{\varepsilon}-\mu_{\varepsilon}\left(u_{\varepsilon}\right)\right]\left(u_{\varepsilon}-\varepsilon\right) \varphi d r d t \\
\quad+\int_{0}^{\infty}(r+\varepsilon)^{N-1} \varphi(r, 0)\left(u_{0}(r)+\varepsilon\right) d r \\
\iint_{\Omega_{T}}(r+\varepsilon)^{N-1}\left[\frac{1}{2} q\left(\left(u_{\varepsilon}\right)^{2}\right)_{r} \varphi_{r}-q_{\varepsilon} u_{\varepsilon} \varphi_{t}\right] d r d t \\
=\iint_{\Omega_{T}}(r+\varepsilon)^{N-1}\left(\beta\left(u_{\varepsilon}\right)-\mu\left(u_{\varepsilon}\right)\right) q u_{\varepsilon} \varphi d r d t \\
\quad+\int_{0}^{\infty}(r+\varepsilon)^{N-1}\left(u_{0}(r)+\varepsilon\right) q_{0}(r) \varphi(r, 0) d r \\
\quad+\varepsilon \iint_{\Omega_{T}}(r+\varepsilon)^{N-1} q\left(\mu\left(u_{\varepsilon}\right)-\beta\left(u_{\varepsilon}\right) e^{-\alpha t} q\right) \varphi d r d t \\
\quad+\iint_{\Omega_{T}} \frac{1}{2}(N-1)\left[\left(\left(u_{\varepsilon}+\varepsilon\right)^{2}\right)_{r}-\left(\left(u_{\varepsilon}\right)^{2}\right)_{r}\right] d r d t .
\end{aligned}
$$


Since $\left|u_{\varepsilon}\right|_{\alpha} \leq K^{*}$ by the Arzela-Ascoli theorem we can extract a subsequence $\left\{u^{\varepsilon_{l}}\right\}$ that converges to an $\alpha$-Hölder continuous function $u(r, t)$ uniformly on compact sets. The corresponding subsequence $\left\{q^{\varepsilon_{l}}\right\}$ satisfies $\left|q^{\varepsilon_{l}}\right|_{\alpha} \leq e^{\alpha T}$. Hence it contains a subsequence $\left\{q^{\varepsilon_{l, k}}\right\}$ that converges weakly to a function $q(r, t),|q|_{\infty} \leq e^{\alpha t}$. We rename both $\left\{u^{\varepsilon_{l, k}}\right\}$ and $\left\{q^{\varepsilon_{l, k}}\right\}$ as $\left\{u_{k}\right\}$ and $\left\{q_{k}\right\}$, respectively. We shall prove that $u^{2}$ is differentiable in $\Omega_{T}$ and that $\left\{\left(u_{k}^{2}\right)_{r}\right\}$ converges pointwise to $\left(u^{2}\right)_{r}$. Then all the integrals will converge in $(2.42)-(2.43)$.

The proof of the differentiability of $u^{2}$ is divided into two cases:

First, if $u\left(r_{0}, t_{0}\right)=\eta_{1}>0$ by the uniform convergence on compact sets we must have that for $k \geq k_{0}, u_{k}(r, t) \leq \frac{\eta}{2}$ in a neighborhood $N$ of $\left(r_{0}, t_{0}\right)$. It follows then by the standard theory of the porous medium equation that $u_{k}$ 's are classical solutions on $N$ and $\left|u_{k}\right|_{2+\alpha} \leq K_{6}$, where $K_{6}$ depends only on $\eta_{1}$ and $N$. Thus there exists a subsequence $\left\{u_{k, n}\right\}$ such that $\left\{\left(u_{k, n}^{2}\right)_{r}\right\}$ converges uniformly to $\left(u^{2}\right)_{r}$ in $N$. In particular $u^{2}$ is differentiable at $\left(r_{0}, t_{0}\right)$.

Second, if $u\left(r_{0}, t_{0}\right)=0$, since $u$ is $\alpha$-Hölder continuous for $\alpha>\frac{1}{2}$ we have

$$
\begin{aligned}
\frac{u^{2}\left(r_{0}+h, t\right)-u^{2}\left(r_{0}, t\right)}{h} & =\frac{u^{2}\left(r_{0}+h, t\right)}{h} \\
& =\left(\frac{u\left(r_{0}+h, t\right)}{h^{\alpha}}\right)^{2} h^{2 \alpha-1} \\
& \leq\left(K^{*}\right)^{2} h^{2 \alpha-1} .
\end{aligned}
$$

Thus $u^{2}$ is differentiable at $\left(r_{0}, t_{0}\right)$ and $\left(u^{2}\right)_{r}\left(r_{0}, t_{0}\right)=0$. The proof of the pointwise convergence of a subsequence of $\left\{\left(u_{k}^{2}\right)_{r}\right\}$ to $\left(u^{2}\right)_{r}$ is the same as the onedimensional case given in [20] and we shall omit it here.

\section{Qualitative behavior of solutions.}

3.1. Populated and unpopulated regions. The existence of the interface that separates the populated region from the unpopulated region will be investigated first.

THEOREM 3. If $u\left(r_{0}, t_{0}\right) \geq \eta>0$ then $u\left(r_{0}, t\right)>0$ for all $t \leq t_{0}$. Thus if a region becomes populated at time $t_{0}$ it remains populated for all later times. In particular the initial region $\left[0, R_{2}\right]$ remains populated for all times (but it might tend to 0 as $t \rightarrow \infty)$.

Proof. Assume first $t_{0}=0$ and $r_{0}>0$. Using the continuity of $u_{0}(r)$ choose $\delta>0$ small, $\delta<r_{0} / 2$, such that $\delta^{2} \leq \frac{\eta}{2}$ and $u_{0}(r) \leq \frac{\eta}{2}$ on $\left(r_{0}-\delta, r_{0}+\delta\right)$. Define $v(r, t)=e^{-k t}\left(\delta^{2}-\left(r-r_{0}\right)^{2}\right)+\frac{\varepsilon}{2}$, over $B=\left(r_{0}-\delta, r_{0}+\delta\right) \times(0, T], k=4 N+M_{0}$. If

$$
\mathscr{L}[z]=z_{t}-z z_{r r}-z_{r}^{2}-\frac{(N-1)}{r+\varepsilon}(z-\varepsilon)+\left(M_{0}+2 N\right)(z-\varepsilon)
$$

we have that $\mathscr{L}\left[u_{\varepsilon}\right]=\left(h+M_{0}+2 N\right)\left(u_{\varepsilon}-\varepsilon\right) \geq 0$ and since $\left|\left(r-r_{0}\right) /(r+\varepsilon)\right| \leq 1$, $e^{-k t} \leq 1$, thus

$$
\begin{aligned}
\mathscr{L}[v] & \leq\left(\delta^{2}-\left(r-r_{0}\right)^{2}\right)\left(4 N+M_{0}-k\right)-4 e^{-k t}\left(r-r_{0}\right)^{2}-\left(M_{0}+2 N\right) \frac{\varepsilon}{2} \\
& \leq 0 .
\end{aligned}
$$


Also

and

$$
v(r, 0)=\delta^{2}-\left(r-r_{0}\right)^{2}+\frac{\varepsilon}{2} \leq \delta^{2}+\frac{\varepsilon}{2} \leq \frac{\eta}{2}+\varepsilon \leq u_{\varepsilon}(r, 0)
$$

$$
v\left(r_{0} \pm \delta, t\right)=\frac{\varepsilon}{2} \leq \varepsilon \leq u_{\varepsilon}\left(r_{0} \pm \delta, t\right) .
$$

The maximum principle now implies that $v(r, t) \leq u(r, t)$ on $\bar{B}=\left[r_{0}-\delta, r_{0}+\delta\right] \times$ $[0, T]$. In particular

$$
u_{\varepsilon}\left(r_{0}, t\right) \geq e^{-k t} \delta^{2}+\frac{\varepsilon}{2} .
$$

Letting $\varepsilon \rightarrow 0$ we obtain $u\left(r_{0}, t\right) \geq \delta^{2} e^{-\left(4 N+M_{0}\right) t}$, for all $t \geq 0$.

If $t_{0}>0$ we use the Hölder continuity of $u$ and the uniform convergence of $u_{\varepsilon}$ to $u$ to find a $\delta$ such that

$$
u_{\varepsilon}\left(r, t_{0}\right) \geq \frac{\eta}{2}+\varepsilon \quad \text { on }\left[r_{0}-\delta, r_{0}+\delta\right] \text {. }
$$

Then the function $v^{\varepsilon}(r, t)=u_{\varepsilon}\left(r, t+t_{0}\right)$ satisfies all the requirements of the previous argument.

If $r=0$ or actually $r \in\left[0, R_{2}\right]$, the result follow directly from the regularity of the porous medium equation with positive initial data.

3.2. Interfaces. We have shown in the proof of Lemma 7 that $\varepsilon \leq u_{\varepsilon}(r, t) \leq 2^{k / m} \varepsilon$ for $r \geq R_{m}$. As $\varepsilon \rightarrow 0$ we obtain that $u(r, t)=0$ for $r \geq R_{m}$. Thus the support of $u(r, t)$ is finite for all $t$. On the other hand, by Theorem 3 we know that once $u$ becomes positive it stays positive for all later times; hence the support of $u(r, t)$ increases with $t$. In particular, if $\operatorname{supp} u_{0}(r)$ is an interval, another application of the maximum principle will show that $\operatorname{supp} u(r, t)$ is also an interval. We state the following result without proof.

THEOREM 4. If $u_{0}(r)>0$ on $\left[0, R_{2}\right], u_{0}(r) \equiv 0$ on $\left[R_{2}, \infty\right)$, then there exists a continuous increasing interface curve $r=\xi(t)$, with $R_{2}=\xi(0)$, separating the populated region $\{(r, t): u(r, t)>0\}$ from the unpopulated region $\{(r, t): u(r, t)=$ $0\}$, i.e., $\operatorname{supp} u(r, t)=[0, \xi(t)]$ or all $t$.

3.3. Localization. We now turn to the question of localization. As $t \rightarrow \infty$, does $\operatorname{supp} u(r, t)$ increase to a limiting domain $[0, L]$ or does $\operatorname{supp} u(r, t)$ increase to $\mathbf{R}^{+}=[0, \infty) ?$

A first observation is that if $\beta(s) \leq \mu(s)-\delta$ for all $s$, then

$$
h(r, t, u)=\beta(u) e^{-\alpha t} q-\mu(u) \leq \beta(u)-\mu(u) \leq \delta<0 .
$$

In this case by the usual comparison arguments $u(r, t)$ is bounded above by the solution $v(r, t)$ of

$$
\begin{aligned}
v_{t} & =v v_{r r}+v_{r}^{2}+(N-1) \frac{v v_{r}}{r}-\delta v, \\
v(r, 0) & =u_{0}(r) .
\end{aligned}
$$

In turn this equation is simplified by letting $t(\tau)=-\frac{1}{\delta} \log (1-\delta \tau)$ and $z(r, \tau)=$ $\frac{1}{1-\delta \tau} v(r, t(\tau))$. Then $z(r, \tau)$ satisfies

$$
\begin{aligned}
z_{\tau} & =z z_{r r}+z_{r}^{2}+(N-1) \frac{z z_{r}}{r}, \\
z(r, 0) & =v(r, 0)=u_{0}(r) .
\end{aligned}
$$


This is a porous medium equation and thus it has finite support for every $\tau$. There exists $L>0$ such that $\operatorname{supp} z(r, \tau) \subseteq[0, L]$ for $0 \leq \tau \leq \frac{1}{\delta}$. Hence $\operatorname{supp} v(r, \tau)=$ $\operatorname{supp} z(r, \tau) \subseteq[0, L]$, because $u(r, t) \leq v(r, t), \operatorname{supp} u(r, t) \subseteq[0, L]$ for all times.

Next, if $h(r, t, u)=\beta(u) e^{-\alpha t} q-\mu(u) \geq 0$, the comparison principle implies that $u(r, t) \geq w(r, t)$, the solution of the porous medium equation

$$
\begin{aligned}
w_{t} & =w w_{r r}+w_{r}^{2}+(N-1) \frac{w w_{r}}{r}, \\
w(r, 0) & =u_{0}(r) .
\end{aligned}
$$

It is known, [23] that $\operatorname{supp} w(r, t) \rightarrow[0, \infty)$ as $t \rightarrow \infty$. Therefore $\operatorname{supp} u(r, t) \rightarrow$ $[0, \infty)$ in this case.

Thus, if the birth module is "clearly" less than the death module, the population will not diffuse further than a fixed interval. On the other hand if $h(r, t, u) \geq 0$, the population will eventually cover all $[0, \infty)$. Unfortunately the condition $\beta(u) \leq$ $\mu(u)-\delta$ is too restrictive and the condition $h \geq 0$ cannot be checked based on the data alone. More general conditions for localization were given in [19] for the onedimensional problem. Those results and examples can be obtained here with only the obvious changes that are omitted.

Let

$$
\begin{array}{cc}
a_{1}=\inf _{0 \leq s \leq M_{1}} \frac{\beta(s)-\alpha}{\beta(s)}, & a_{2}=\sup _{0 \leq s \leq M_{1}} \frac{\beta(s)-\alpha}{\beta(s)}, \\
b_{1}=\inf _{0 \leq s \leq M_{1}} \frac{\mu(s)}{\beta(s)}, & b_{2}=\sup _{1 \leq s \leq M_{1}} \frac{\mu(s)}{\beta(s)} .
\end{array}
$$

TheOREM 5. (i) Assume $a_{2}<b_{1}$ and $0<\bar{m} \leq q_{0}(r) \leq c_{1}$ for some $c_{1} \in\left(a_{2}, b_{1}\right)$. Then there exists $L>0$ such that the solution $u(r, t)$ is identically 0 outside $[0, L]$ for all $t$.

(ii) Assume $b_{2}<a_{1}$ and $c \leq q_{0}(r) \leq 1$ for some $c \in\left(b_{2}, a_{1}\right)$. Then the support of $u(r, t)$ tends to $[0, \infty)$ as $t \rightarrow \infty$.

3.4. Examples. Let $\beta^{*}=\sup _{0 \leq s \leq M_{1}} \beta(s), \beta_{*}=\inf _{0 \leq s \leq M_{1}} \beta(s)$ and $\mu^{*}, \mu_{*}$ defined similarly.

If $\beta^{*} \leq \mu_{*}+\alpha$ and $q_{0}(r) \leq c_{1}$ for some $c_{1} \in\left(\left(\beta^{*}-\alpha\right) / \beta^{*}, \mu_{*} / \beta^{*}\right)$, then Theorem 5 implies that $u(r, t)$ is localized. On the other hand if $\beta_{*} \geq \mu^{*}+\alpha$ and $c \leq q_{0}(r) \leq 1$ for some $c \in\left(\mu^{*} / \beta_{*},\left(\beta_{*}-\alpha\right) / \beta_{*}\right)$, then supp $u(r, t)$ tends to $[0, \infty)$ as $t \rightarrow \infty$. These results were first conjectured by Gurtin [13], for $\beta$ and $\mu$ constants.

\section{REFERENCES}

[1] D. G. Aronson, The porous medium equation, CIME Lectures Montecatini Terme, 10-18 (June 1985)

[2] D. G. Aronson, Regularity properties of flows through porous media, SIAM J. Appl. Math. 17 461-467 (1969)

[3] D. G. Aronson, Regularity properties of flows through porous media: a counterexample, SIAM J. Appl. Math. 19, 299-307 (1970)

[4] $\mathrm{Ph}$. Benilam, $A$ strong regularity Lp for solutions of the porous media equations, Contributions to Nonlinear Partial Differential Equations, C. Bardos, A. Damlamian, J. I. Diaz and J. Hernandez, Editors, London, 1983, pp. 39-58 
[5] L. A. Caffarelli and A. Friedman, Continuity of the density of a gas flow in a porous medium, Trans. Amer. Math. Soc. 252, 99-113 (1979)

[6] L. A. Caffarelli and A. Friedman, Regularity of the free boundary of a gas flow in an n-dimensional porous medium, Indiana Univ. Math. J. 29, 361-391 (1980)

[7] E. A. Carl, Population control in arctic ground squirrels, Ecology 52, 395-413 (1971)

[8] D. G. Aronson and L. A. Caffarelli, Optimal regularity for one-dimensional porous medium flow, Rev. Math. Iberoamericana 2, 357-366 (1986)

[9] Gabriella Di Blasio, Nonlinear age-dependent population diffusion, J. Math. Biology 8, 265-284 (1979)

[10] E. di Benedetto, Continuity of weak solutions to a general Porous medium equation, Indiana Univ. Math. J. 32, 83-118 (1983)

[11] Maria Garroni and Michel Langlais, Age dependent population diffusion with external constraints, J. Math. Biol. 14, 77-94 (1982)

[12] W. S. C. Gurney and R. M. Nibset, The regulation of inhomogeneous populations, J. Theoretical Biology 52, 441-457 (1975)

[13] M. E. Gurtin, Some questions and open problems in continuum mechanics and population dynamics, J. Differential Equations 48, 293-312 (1983)

[14] M. E. Gurtin and R. C. MacCamy, Diffusion models for age-structured populations, Math. Biosciences 54, 49-59 (1981)

[15] M. E. Gurtin and R. C. MacCamy, Nonlinear age-dependent population dynamics, Arch. Rational Mech. Anal. 54, 281-300 (1974)

[16] M. E. Gurtin and R. C. MacCamy, On the diffusion of biological populations, Math. Biosciences 33, 35-49 (1977)

[17] M. E. Gurtin and R. C. MacCamy, A population model with nonlinear diffusion, J. Differential Equations 39, 52-72 (1981)

[18] M. E. Gurtin and R. C. MacCamy, Some simple models for nonlinear age dependence population dynamics, Math. Biosciences 43, 199-211 (1979)

[19] Gaston E. Hernandez, Dynamics of populations with age dependence and diffusion: localization, Appl. Anal. 29, 143-163 (1988)

[20] Gaston E. Hernandez, Existence of solutions in a population dynamic problem, Quart. Appl. Math. 43, 509-521 (1986)

[21] Gaston E. Hernandez, The Hölder property in some degenerate parabolic problems, J. Differential Equations 65, 240-249 (1986)

[22] Gaston E. Hernandez and Ioannis Roussos, The Hölder exponent for radially symmetric solutions of porous medium type equations, to appear, in Canad. J. Math., 1990.

[23] A. S. Kalashnikov, On the occurrence of singularities in the solutions of the equation of nonstationary filtration, Zh. Vychisl. Mat. i Mat. Fiz. 14, 891-905 (1974)

[24] Michel Langlais, A nonlinear problem in age dependent population diffusion, Preprint, 1983

[25] O. A. Oleinik and S. N. Kruzhkov, Quasilinear second order parabolic equations with many independent variables, Russian Math. Surveys, 16, 106-146 (1961)

[26] L. A. Peletier, The porous media equation, Applications of Nonlinear Analysis in the physical Sciences, H. Amann et al. editors, Pitman, London, 1981, pp. 229-241

[27] Paul Sacks, Existence and regularity of solutions of inhomogeneous porous medium type equations, T.S.R. No. 2214 M.R.C. Madison, Wisconsin, 1985

[28] J. G. Skellam, Random dispersal in theoretical populations, Biometrika 38, 196-218 (1951)

[29] J. L. Vazquez, La ecuacion de los medios porosos., Actas 1 ra Jornadas de Mecanica y Fisica Matematicas, El Escorial, Sept. 1983 